\title{
Magnetoelastic Interactions in the Hexagonal Belorizky Model
}

\author{
Z. Bak \\ Institute of Physics, Pedagogical University of Czeștochowa, Czeștochowa, Poland
}

Z. Naturforsch. 41 a, $1347-1351$ (1986); received May 22, 1986

\begin{abstract}
The theory of dynamical magnetoelasticity in the hexagonal Belorizky model is presented in this paper. We show that the interaction of spins with lattice vibrations gives rise to multiaxis anisotropic biquadratic exchange. It is shown that this anisotropy favours some directions and can stabilize three-sublattice ordering of the system. Formulae for low-temperature spin wave spectra were obtained within the framework of the Dyson-Maleev transformation.
\end{abstract}

\section{Introduction}

Spin models with anisotropic exchange receive great attention in theoretical as well as in experimental research because they display a behaviour which is qualitatively different from that of standard models with isotropic Heisenberg exchange. Moreover, in low-dimensional spin systems a small anisotropy in higher dimension is necessary to obtain finite magnetization.

The aim of this paper is to study the properties of the anisotropic model proposed by Belorizky et al. [1] adapted to the case of a hexagonal, elastic lattice crystal. The Belorizky model has been recently an object of intensive studies [2-7]. In this paper we show that the interaction of spins with ion displacements leads to anisotropic biquadratic exchange between neighbouring spins. Despite the fact that the initial Hamiltonian is orientationally isotropic [1] (i.e. the magnetization is always directed along the direction of the applied magnetic field), the effective Hamiltonian is anisotropic. The terms of multiaxis anisotropic biquadratic exchange are of the easy axis type and tend to align the spins along the prefered axes.

We have concentrated our attention on quasi 2-D crystals of hexagonal symmetry with antiferromagnetic nearest neighbour exchange. It can be easily shown that in this case a three-sublattice $120^{\circ}$ spin structure can be energetically favourable [8]. As it was shown by Meier [9], long range order cannot appear if the exchange interaction is restricted to isotropic exchange. We show that the anisotropic

Reprint requests to Dr. Z. Bak, Institute of Physics, Pedagogical University of Czeștochowa, al. Zawadzkiego 13. biquadratic exchange resulting from thermal oscillations of magnetic ions can stabilize such a threesublattice $120^{\circ}$ spin order.

In Chapt. 3, within the Dyson-Maleev [10] transformation from spin to Bose magnon creation and destruction operators we study the spin wave spectra of our model in case of a three-sublattice $120^{\circ}$ spin structure.

\section{Hamiltonian}

Let us consider an array of localized spins forming a simple hexagonal lattice. The interaction between two spins neighbouring in the " $v$ " direction is described by the form [2]

$$
H_{n}^{v}=J^{v}\left[S_{n} S_{n+e_{v}}+\eta_{v} S_{n}^{v} S_{n+e_{v}}^{v}\right],
$$

where $S_{n}^{v}=\boldsymbol{S}_{n} \bar{e}_{v}$ ( $\bar{e}_{v}$ is a unit vector oriented along the " $v$ " direction). The spin with index $n+e_{v}$ is the nearest neighbour of spin with index $n$ located at the position

$$
R_{n+e_{v}}=R_{n}+\bar{a}_{v} \bar{e}_{v} .
$$

( $\bar{a}_{v}$ is the lattice spacing in the " $v$ " direction).

An anisotropic interaction among spins of the form (1), invariant with respect to rotation around the axis through the interacting ions, has first been postulated by Van Vleck [11]. The bond of the form (1) gives a good description of interaction of $\mathrm{Co}^{2+}$ ions (with $S=1 / 2$ ) in $\mathrm{KCoF}_{3}$ [12] and $\mathrm{Ni}^{2+}$ ions (with $S=1$ ) in $\mathrm{NiO}$ [13].

As it has been shown by J. Wolf [14], the interaction (1) represents the most general form of interaction between two weakly-coupled Kramer's doublets when their bond is invariant under $\mathrm{C}_{4 \mathrm{v}}, \mathrm{C}_{4 \mathrm{~h}}$, 
$\mathrm{D}_{4}, \mathrm{D}_{4 \mathrm{~h}}, \mathrm{C}_{3 \mathrm{v}}, \mathrm{C}_{3 \mathrm{~h}}$ symmetry groups. Magnetic systems of $\mathrm{C}_{4 \mathrm{v}}, \mathrm{C}_{4 \mathrm{~h}}, \mathrm{D}_{4}, \mathrm{D}_{4 \mathrm{~h}}$ symmetry concerning cubic crystals have been an object of intensive studies in a series of papers [1-6]. In the following chapters we will consider a case of $S=1$ system with trigonal symmetry of magnetic bonds.

After carrying out summation over all possible orientations of neighbouring spins we obtain the Hamiltonian of the magnetic system in the form

$$
H_{0}=\sum_{n, v} J^{r}\left[S_{n} S_{n+e_{v}}+\eta_{v} \cdot S_{n} \cdot S_{n+e_{v}}\right]
$$

$v=a, b, \zeta, c=$ principal axis of a simple hexagonal lattice. By writing the Hamiltonian in the form (3) we have neglected completely any single ion anisotropy of our system. Due to the hexagonal symmetry of the considered model we have: $J^{a}=J^{b}=$ $J^{\zeta}=J^{0}$ (the intraplanar exchange integral) and $J^{c}=J^{y}$ (the interplanar exchange integral). Similarly $\eta_{a}=\eta_{b}=\eta_{\zeta}=\eta_{0}$ and $\eta_{c}=\eta_{y}$. The global coordination axes " $y$ " and " $z$ " are choosen parallel to the " $c$ " and " $a$ " axes, respectively.

In case of $\eta_{0}=1$ and $\eta_{y} \neq 1$ the Hamiltonian [3] describes magnetic interactions in compounds of the $\mathrm{ABX}_{3}$ type $(\mathrm{A}=\mathrm{Cs}, \mathrm{Rb} ; \mathrm{B}=\mathrm{Fe}, \mathrm{Ni} ; \mathrm{X}=\mathrm{Cl}, \mathrm{Br})$; for details see [15-19]. In these compounds magnetic ions form triangularity arranged linear chains with moments lying in a plane perpendicular to the caxis.

Although the interaction [1] is anisotropic the resulting Hamiltonian [3] is isotropic (for $J^{0}=J^{y}$ ), i.e. in case of ferromagnetic ground state, the magnetization is always oriented along the direction of the applied magnetic field. It $s$ worthwile to note that the Hamiltonian [3] cannot be reduced to the isotropic Heisenberg model.

So far, we have considered that the spins are located at the sites of the immobile lattice. Now we introduce coupling between magnetic and lattice degrees of freedom of the system. The integrals $J^{0}$ and $J^{y}$ are assumed to depend on the positions $R_{\mathrm{n}}$ of magnetic ions:

$$
R_{n}=R_{n}^{0}+x_{n}=x_{n}+\left\langle R_{n}\right\rangle .
$$

We expand the exchange integrals in power series of $x_{n}$, the thermal displacements of ions from the equilibrium position $R_{n}^{0}=R_{n}^{0}(T)$, and retain only the first and second terms of the expansion. The first term of the expansion describes spin interactions between spins on the rigid lattice. The second is an interaction between spins and lattice vibrations, assumed to be harmonic.

Finally, the Hamiltonian of the system, with lattice vibrations taken into account, can be written as (for details see [6], [20-21])

$$
\begin{aligned}
H=H_{0} & \sum_{n} p_{n}^{2} /\left(2 m_{n}\right)+\sum_{n r r}\left(x_{n}^{r}-x_{n+e_{v}}^{r}\right)^{2} /\left(2 \varepsilon_{v}\right)+ \\
& +\sum_{n r r} \Delta J_{n, r}^{r}\left[S_{n} S_{n+e_{v}}+\eta_{v} S_{n}^{v} S_{n+e_{v}}^{r}\right]\left[x_{n}^{r}-x_{n+e_{v}}^{r}\right],
\end{aligned}
$$

where $\Delta J_{n, r}^{v}=\partial J^{v} / \partial x_{n}^{r}, \quad v=a, b, \zeta, \quad r=x, y, z$ and $x_{n}^{r}$ is the time dependent part of the displacement of an ion from the equilibrium position. The Hamiltonian (4) contains terms which describe: exchange between the nearest neighbours, the kinetic energy of oscillating ions, the energy of the harmonic lattice and the exchange modulations by lattice vibrations, respectively.

We assume $\eta_{v}$ to be small (typically $\eta \sim 0.1,[12]$ ). Thus we have neglected in (4) terms which are proportional to $\partial \eta_{v} / \partial x_{n}^{r}$. Now we will focus our attention on the magnetoelastic part of the Hamiltonian (4). $\Delta J_{n, r}^{v}$ can be written in the form

$\Delta J_{n, r}^{r}=\frac{\partial J^{r}\left(R_{n}-R_{n+e_{v}}\right)}{\partial x_{n}^{r}}=\frac{\partial J^{r}\left(\varrho_{n}^{r}\right)}{\partial \underline{\varrho}_{n}^{r}} \frac{\partial \varrho_{n}^{r}}{\partial x_{n}^{r}}$,

where

$$
\begin{aligned}
\varrho_{n}^{v} & =\left|R_{n}-R_{n+e_{v}}\right| \\
& =\left[\left(R_{n}^{0}-R_{n+e_{v}}^{0}+x_{n}^{v}-x_{n+e_{v}}^{v}\right)^{2}+\left(x_{n}^{\perp v}-x_{n+e_{v}}^{\perp v}\right)^{2}\right]^{\frac{1}{2}} .
\end{aligned}
$$

$R_{n}^{0}$ is the equilibrium position of an ion, $x_{n}^{v}=\bar{x}_{n} \cdot \bar{e}_{v}$, $x_{n}^{\perp v}$ is orthogonal to the $x_{n}^{r}$ part of the displacement of an ion from the equilibrium position. From (6) we have

$$
\left.\frac{\partial\left(\varrho_{n}^{v^{\prime}}\right)}{\partial x_{n}^{r^{r}}}\right|_{x_{n}=0}=A^{v} \delta_{r, v^{\prime}} .
$$

Thus, the last term of the Hamiltonian (4) can be written in the form

$$
\begin{aligned}
& \sum_{n, r} \Delta J_{n, r}^{r}\left[S_{n} S_{n+e_{v}}+\eta_{v} S_{n}^{v} S_{n+e_{v}}^{v}\right]\left[x_{n}^{r}-x_{n+e_{v}}^{r}\right] \\
& \quad=\sum_{n r} \Delta J^{v}\left[S_{n} S_{n+e_{v}}+\eta_{v} S_{n}^{r} S_{n+e_{v}}^{r}\right]\left[x_{n}^{r}-x_{n+e_{v}}^{v}\right]=H_{\mathrm{el}},
\end{aligned}
$$

with

$$
\Delta J^{v}=A^{r} \cdot\left(\partial \cdot J^{r}\right) /\left(\partial \underline{Q}_{n}^{v}\right) .
$$

As we can see, the magnetic degrees of freedom of our system are coupled to that part of the displace- 
ment of an ion which is parallel to the vector $R_{n}^{0}-R_{n+e_{v}}^{0}$ joining the interacting ions in the rigid lattice. If we introduce the auxiliary quantity

$$
d_{n, r}^{r}=x_{n}^{r}-x_{n+e_{v}}^{r},
$$

being the change of distance of interacting ions, $H_{\mathrm{el}}$ can be written in the form

$$
H_{\mathrm{el}}=\sum_{n r v} \Delta J^{v}\left[S_{n} S_{n+e_{v}}+\eta_{v} S_{n}^{v} S_{n+e_{v}}^{v}\right] \cdot B_{r}^{v} \cdot d_{n, r}^{v}
$$

with $B_{r}^{r}=\left(\partial x_{n}^{r}\right) /\left(\partial x_{n}^{v}\right)$. The explicit values of $B_{r}^{v}$ are given by (A.1) in the Appendix. Taking (10) and (11) into account, the Hamiltonian (4) takes the form

$$
\begin{aligned}
H=H_{0} & +\sum_{n r v}\left(d_{n, r}^{v}\right)^{2} /\left(2 \varepsilon_{v}\right)+\sum_{n} p_{n}^{2} /\left(2 m_{n}\right) \\
& +\sum_{n r v} \Delta J^{v}\left[S_{n} S_{n+e_{v}}+\eta_{v} S_{n}^{v} S_{n+e_{v}}^{v}\right] \cdot B_{r}^{v} \cdot d_{n, r}^{v} .
\end{aligned}
$$

Making a transformation of the type

$$
\begin{aligned}
d_{n, r}^{v}= & D_{n, r}^{v}-\Delta J^{v} \varepsilon_{v} \\
& \cdot\left[S_{n} S_{n+e_{v}}+\eta_{v} S_{n}^{v} S_{n+e_{v}}^{v}\right] B_{r}^{v}
\end{aligned}
$$

(for details see [6] and [22]), the Hamiltonian (12) can be written in the form

$$
\begin{aligned}
H= & H_{0}+\sum_{n} p_{n}^{2} /\left(2 m_{n}\right)+\sum_{n r v}\left(D_{n, r}^{v}\right)^{2} /\left(2 \varepsilon_{v}\right) \\
& +\sum_{n v} J_{2}^{r}\left[S_{n} S_{n+e_{v}}+\eta_{v} S_{n}^{v} S_{n+e_{v}}^{v}\right]^{2}=H_{\mathrm{L}}+H_{M},
\end{aligned}
$$

with $J_{2}^{v}=\left(\Delta J^{v}\right)^{2} \cdot \varepsilon_{v}\left(\sum_{r} B_{r}^{v}\right) / 2$.

In this way we have decoupled our system into two subsystems: spins and elastic lattice with no coupling between then. Next we will focus our attention on the magnetic properties of the considered system, which can be described by the spin dependent part of the Hamiltonian (14)

$$
\begin{aligned}
H_{\mathrm{M}}= & \sum_{n v} J^{v}\left[S_{n} S_{n+e_{v}}+\eta_{v} S_{n}^{v} S_{n+e_{v}}^{v}\right] \\
& +\sum_{n r} J_{2}^{v}\left[S_{n} S_{n+e_{v}}+\eta_{v} S_{n}^{v} S_{n+e_{v}}^{v}\right]^{2} .
\end{aligned}
$$

The first term of (15) describes the spin interactions on the rigid lattice, the second represents the anisotropic biquadratic exchange being an effect of thermal vibrations. The Hamiltonian (15) has a form as if instead of the bond (1) we would have used a bond of the form

$$
\begin{aligned}
H_{n}^{0}= & J^{v}\left[S_{n} S_{n+e_{v}}+\eta_{v} S_{n}^{v} S_{n+e_{v}}^{v}\right] \\
& +J_{2}^{v}\left[S_{n} S_{n+e_{v}}+\eta_{v} S_{n}^{v} S_{n+e_{v}}^{v}\right]^{2}
\end{aligned}
$$

and would then have constructed the analogue to (3). As it has been shown in [20], $J_{2}^{r}$ can be of the order of $J_{2}^{r} \sim 0.1 J^{r}$. It results from (15) that the dynamical interactions give rise to anisotropic biquadratic exchange among spins. It is a well known fact that the biquadratic exchange (independently on its origin) gives a nontrivial contribution only for $S \geqq 1$. We assume this condition to be satisfied. The system described by the Hamiltonian (3) is isotropic while the system described by the Hamiltonian (15) is no longer isotropic. It can easily be shown that our system possesses preferable directions of easy (for $J_{2}^{v} \eta_{v}<0$ ) or difficult (for $J_{2}^{v} \eta_{v}>0$ ) magnetization. They are the principal axes of the crystal. For $\eta_{v}=0$ we have the well known results for the case of isotropic biquadratic exchange (see e.g. [23-25]).

In the following we will study the general case of a 3-D spin system described by the Hamiltonian (15). We will limit ourselves to the case of $S=1$, simple hexagonal lattice with three-sublattice antiferromagnetic ground state.

\section{Spin Waves in Triangular $120^{\circ}$ Antiferromagnet}

In this chapter we will consider the case of antiferromagnetic arrangements of spins. Since our system has a hexagonal symmetry with $J^{0}>J^{y}$, the spins are arranged in hexagonal planes. When the exchange integrals are positive, the moments lying in the hexagonal plane form a triangular spin structure [8]. In the following we assume that the low temperature phase of the system has an antiferromagnetic $120^{\circ}$ spin structure. The lattice is decomposed on three geometrically identical interpenetrating sublattices. Each spin is surrounded by spins on the nearest neighbour sites belonging to different sublattices. From (15) results that the magnetizations of sublattices are oriented along the axes of easy magnetization.

We are interested in the low-temperature properties of the system described by the Hamiltonian (15). For this purpose it suffices to use the DysonMaleev representation of spin operators (see e.g. [10]):

$$
S^{+}=(2 S)^{\frac{1}{2}} a, \quad S^{-}=(2 S)^{\frac{1}{2}} a^{+}, \quad S^{z}=S-a^{+} a .
$$


When we apply the transformation (17) to our system denoting by $x_{a}^{+}$and $x_{a}$ the Bose magnon creation and destruction operators on the sublattices $\alpha$, $\beta$ and $\gamma$, respectively, the bilinear part of bosonic equivalent Hamiltonian takes the form

$$
\begin{aligned}
H_{2}= & \sum_{k, a} w_{k} x_{a, k}^{+} x_{a, k} \\
& +\sum_{a, k} \beta_{k}\left[x_{a, k} x_{a,-k}+\text { h.c. }\right] \\
& +\sum_{k, a=b} \delta_{k} x_{a}^{+} \cdot k x_{b, k} \\
& +\sum_{k, a=b} \Phi_{k}\left[x_{a, k}^{+} x_{b, k}+\text { h.c. }\right]
\end{aligned}
$$

$x_{a, k}^{+}$and $x_{a, k}$ are the Fourier transforms of Bose magnon creation and destruction operators $(a=$ $\alpha, \beta, \gamma)$. The coefficients $w_{k}, \beta_{k}, \delta_{k}$ and $\Phi_{k}$ are given by (A. 2) in the Appendix. The Hamiltonian (18) can easily be diagonalized by linear transformation of the form [10]

$$
\lambda_{a, k}=\sum_{b}\left[U_{b, k}^{a} x_{b, k}^{+}+V_{b, k}^{a} x_{b, k}\right] .
$$

$U_{b, k}^{a}$ and $V_{b, k}^{a}$ are real functions related by

$$
\sum_{b}\left[\left(U_{b, k}^{a}\right)^{2}-\left(V_{b, k}^{a}\right)^{2}\right]=1
$$

because the new creation and destruction operators must satisfy the commutation rules for Bose operators

$$
\left[\lambda_{a, k}^{+}, \lambda_{b, k}\right]=-\delta_{a, b}
$$

After diagonalization, the Hamiltonian (18) takes the form

where

$$
H_{2}=\sum_{a, k} E_{k}^{a} \lambda_{a, k}^{+} \lambda_{a, k}
$$

$$
E_{k}^{1}=\left[\left(w_{k}+2 \Phi_{k}\right)^{2}-\left(\beta_{k}+2 \delta_{k}\right)^{2}\right]^{\frac{1}{2}},
$$

and

$$
E_{k}^{2,3}=\left[\left(w_{k}-2 \Phi_{k}\right)^{2}-\left(\beta_{k}-2 \delta_{k}\right)^{2}\right]^{\frac{1}{2}} .
$$

The expressions for the coefficients $U_{b, k}^{a}$ and $V_{b, k}^{a}$ of the Bogolyubov transformation (19) that diagonalizes the Hamiltonian (18) are given by formulae (A.3) in the Appendix. The Eqs. (23) give us the spin wave spectra of our three-sublattice antiferromagnetic system.

\section{Summary}

We have performed a systematic treatment of a spin system of hexagonal symmetry with anisotropic Heisenberg exchange between the nearest neighbours. In our considerations we have assumed a twofold dependence of the exchange integrals on temperature, first via the thermal expansion and second via the thermal oscillations of ions. The oscillation contribution to the exchange constant is the cause of the directional anisotropy of the system.

Despite of the small (compared to bilinear exchange) energy of the anisotropic biquadratic exchange the accompanying changes in the magnetic system are of importance: biquadratic exchange favours some directions of magnetization and thus prevents frustration of the spin structure. There is an experimental evidence of such a behaviour in cubic crystals. As is was found in CeAs [26] (where the exchange interactions are of Belorizky type), the quadrupolar interactions dominate the $[1,1,1]$ direction predicted by the crystal field and slightly favour the $[0,0,1]$ alignment of spins.

Stabilization of some directions of magnetization prevents frustration of spin structure. This is especially important in hexagonal antiferromagnets with triangular spin structure. As it was shown by Meier [9], in such a system with isotropic exchange long range order cannot appear. Frustrated spin structure with short range triangular order is energetically favourable. Such a (magnetic liquid like) behaviour has been observed recently in $\mathrm{CsVCl}_{3}$ and $\mathrm{VX}_{2}(\mathrm{X}=\mathrm{Cl}, \mathrm{Br}$ and $\mathrm{I})$ compounds of hexagonal symmetry [27]. Thus, if long range triangular ordering exists it must be stabilized by anisotropic interactions.

In the Hamiltonian (3) we have neglected single ion anisotropy of our system. It is well known fact that single ion anisotropy can confine spins to a plane, but in case $S=1$ the spin system cannot produce prefered axes of hexagonal symmetry. Thus the effect predicted by us cannot be explained in terms of single ion anisotropy.

The model under consideration is expected to have applications in the description of low-dimensional systems of hexagonal symmetry. $\mathrm{RbFeCl}_{3}$ like compounds [28] can serve as examples. 


\section{Appendix}

$B_{z}^{a}=1, B_{z}^{b}=B_{z}^{c}=-\frac{1}{2}, B_{x}^{a}=-B_{x}^{b}=\sqrt{3} / 2, B_{y}^{c}=1$,

$B_{r}^{v}=0$ for all other values of $r$ and $v$.

$\beta_{k}=S^{3} \frac{1}{8}\left(12+12 \eta+5 \eta^{2}\right)\left[J_{2}^{0}(0)+\frac{1}{2} J_{2}^{y}(0)\right]$,

$w_{k}=2 \beta_{k}+(2+\eta) S\left[J_{1}^{0}(0)-J_{2}^{0}(0)(1+\eta) S^{2}\right]$

$$
\begin{aligned}
& +\frac{4}{3} S\left[J_{Y}^{y}(0)-J_{2}^{y}(0)\right] \\
& +\frac{1}{2} S^{3}\left(S-\frac{1}{2} \eta\right) J_{2}^{0}(0),
\end{aligned}
$$

$\Phi_{k}=-\frac{1}{12}(14+3 \eta)\left[J_{1}^{0}(k)-J_{2}^{0}(k)(1+\eta) S^{2}\right]$

$\cdot S-\frac{1}{6} S(3+2 \eta)\left[J_{1}^{y}(k)-J_{2}^{y}(k)\right]$

$-\frac{1}{16} S^{3}(2+3 \eta) J_{2}^{0}(k)$

$-\frac{1}{8} S^{3}\left(12+12 \eta+\eta^{2}\right) J_{2}^{0}(k)-S^{3} J_{2}^{y}(k) / 2$,

$\delta_{k}=\Phi_{k}+2 S(1+\eta)\left[J_{1}^{y}(k)-J_{2}^{y}(k)\right] / 3$

$+2 S\left[J_{1}^{0}(k)-J_{2}^{0}(k)\right](1+\eta)$.

$U_{1}^{a}=V_{2}^{a}=\left[\frac{w_{k}+2 \Phi_{k}+E_{1}}{6 E_{1}}\right]^{\frac{1}{2}}$,

[1] E. Belorizky, R. Casalegno, and P. Fries, Phys. Stat. Sol. 77 b, 495 (1976).

[2] E. Belorizky, R. Casalegno, P. Fries, and J. Niez, J. Physique 39, 776 (1978).

[3] M. J. Skrinjar, D. V. Kapor, and J. P. Setrajcic, Phys. Stat. Sol. 103 B, 559 (1981).

[4] M. J. Skrinjar, D. V. Kapor, and J. P. Setrajcic, Phys. Stat. Vol. 117 b, K9 (1983).

[5] Z. Bak, Phys. Stat. Sol. 117 B, K 19 (1983).

[6] Z. Bak, J. Mag. Mag. Mat. 44, 254 (1984).

[7] E. Belorizky, R. Casalegno, and J. Sivardiere, J. Mag. Mag. Mat. 15- 18, 309 (1980).

[8] E. F. Bertaut, J. Phys. Chem. Sol. 21, 295 (1961).

[8a] F. Matsubara, J. Phys. Soc. Japan 51, 2424 (1982).

[9] R. J. Meier, Phys. Lett. 107 A, 275 (1985).

[10] S. V. Tyablikov, Methods in the Quantum Theory of Magnetism, Plenum Press, New York 1967.

[11] J. H. Van Vleck, Phys. Rev. 52, 1178 (1937).

[12] W. J. L. Buyers, T. M. Holden, E. C. Svensson, E. C. Cowley, and H. T. Hutchings, J. Phys. C4, 2135 (1971).

[13] J. Kanamori in "Magnetism", Vol. I, p. 164, G. T. Rado, H. Suhl, eds., Academic Press, New York 1963.

$$
\begin{aligned}
& U_{2}^{a}=-V_{1}^{a}=\frac{\beta_{k}+2 \delta_{k}}{\left[6 E_{1}\left(w_{k}+2 \Phi_{k}+E_{1}\right)\right] \frac{1}{2}}, \\
& U_{3}^{a}=V_{4}^{a}=\left[\frac{\beta_{k}+2 \delta_{k}}{4 E_{2}}\right]^{\frac{1}{2}}, \quad a=2,3, \\
& \begin{aligned}
U_{4}^{a} & =-V_{3}^{a}=\frac{k-k}{\left[4 E_{1}\left(w_{k}-\Phi_{k}+E_{1}\right)\right]^{\frac{1}{2}}}, U_{3,4}^{1}=V_{3,4}^{1}=0 \\
U_{5}^{1} & =-V_{6}^{1}=-2 V_{6}^{2,3}=2 U_{5}^{2,3}=\left[\frac{w_{k}-\Phi_{k}+E_{2}}{3 E_{2}}\right]^{\frac{1}{2}}, \\
V_{5}^{1} & =2 V_{5}^{2,3}=-U_{6}^{1}=-2 U_{6}^{2,3} \\
& =\frac{\left[3 E_{1}\left(w_{k}-\Phi_{k}+E_{1}\right)\right]^{\frac{1}{2}}}{\beta_{k}-\delta_{k}} \\
E_{1} & =E_{k}^{1}, \quad E_{2}=E_{k}^{2,3} \text { of }(23) .
\end{aligned}
\end{aligned}
$$

[14] W. P. Wolf, J. Physique 32, C1 (1971).

[15] N. Suzuki, J. Phys. Soc. Japan 52, 1002 (1983).

[16] W. B. Yelon and D. E. Cox, Phys. Rev. B 7, 1024 (1973).

[17] W. B. Yelon and D. E. Cox, Phys. Rev. B6, 204 (1972).

[18] M. Eibschutz and M. E. Lines, Phys. Rev. B 11, 4583 (1975).

[19] M. Eibschutz, M. E. Lines, and R. C. Sherwood, Phys. Rev. B 11, 4595 (1975).

[20] K. N. Shrivastava, Phys. Lett. 56 A, 399 (1976).

[21] D. C. Mattis and T. D. Schultz, Phys. Rev. 129, 175 (1963).

[22] There is an obvious mistake in Eq. (5) of [6]. The $J_{\perp}$ should be replaced by $\varepsilon \Delta J$.

[23] T. Moriya, Phys. Rev. 120, 90 (1960).

[24] R. Micnas, J. Phys. C9, 3307 (1975).

[25] Z. Bak, Z. Naturforsch. 36 A, 797 (1981).

[26] B. Hälg and A. Furer, J. Appl. Phys. 55, 1860 (1984).

[27] K. Hirakawa, H. Ikeda, H. Kadowaki, and K. Ubukoshi, J. Phys. Soc. Japan 52, 2881 (1993).

[28] N. Suzuki, J. Phys. Soc. Japan 52, 3907 (1983). 